\title{
BMJ Open A qualitative study exploring how Somali women exposed to female genital mutilation experience and perceive antenatal and intrapartum care in England
}

\author{
Jordan M Moxey, Laura L Jones
}

To cite: Moxey JM, Jones LL. A qualitative study exploring how Somali women exposed to female genital mutilation experience and perceive antenatal and intrapartum care in England. BMJ Open 2016;6:e009846. doi:10.1136/bmjopen-2015009846

- Prepublication history for this paper is available online. To view these files please visit the journal online (http://dx.doi.org/10.1136/ bmjopen-2015-009846).

Received 27 August 2015 Revised 10 November 2015 Accepted 3 December 2015

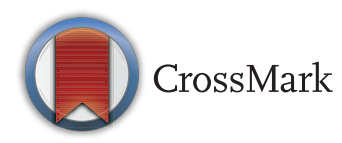

Institute of Applied Health Research, University of Birmingham, Birmingham, UK

\section{Correspondence to} Jordan Moxey; JMM147@student.bham.ac. uk

\section{ABSTRACT}

Objectives: To explore how Somali women exposed to female genital mutilation experience and perceive antenatal and intrapartum care in England. We explored women's perceptions of deinfibulation, caesarean section and vaginal delivery; their experiences of care during pregnancy and labour; and factors that affect ability to access these services, in order to make recommendations about future practice.

Design: A descriptive, exploratory qualitative study using face-to-face semistructured interviews. Interviews were audio-recorded, transcribed and data were analysed using a thematic approach. An interpreter was used when required $(n=3)$.

Setting: Participants recruited from 2 community centres in Birmingham, England.

Participants: Convenience and snowball sample of 10 Somali women resident in Birmingham, who had accessed antenatal care services in England within the past 5 years.

Results: 3 core themes were interpreted: (1) Experiences of female genital mutilation during life, pregnancy and labour. Female genital mutilation had a significant physical and psychological impact, influencing decisions to undergo deinfibulation or caesarean section. Women delayed deinfibulation until labour to avoid undergoing multiple operations if an episiotomy was anticipated. (2) Experience of care from midwives: Awareness of female genital mutilation from midwives led to open communication and stronger relationships with women, resulting in more positive experiences. (3) Adaptation to English life: Good language skills and social support networks enabled women to access these services, while unfavourable social factors (eg, inability to drive) impeded.

Conclusions: Female genital mutilation impacts Somali women's experiences of antenatal and intrapartum care. This study suggests that midwives should routinely ask Somali women about female genital mutilation to encourage open communication and facilitate more positive experiences. As antenatal deinfibulation is unpopular, we should consider developing strategies to promote deinfibulation to

\section{Strengths and limitations of this study}

- This descriptive, exploratory qualitative study is the first, as far as the authors are aware, to explore how Somali women exposed to female genital mutilation experience and perceive antenatal and intrapartum care in England.

- It is often difficult for non-community members to engage the Somali community in research but we were able to successfully recruit a small number of women who were willing to discuss this important and sensitive issue with the researchers.

- The use of lay interpreters may have promoted misinterpretation of questions and responses during interview but overall, appeared to facilitate the development of rapport, put participants at ease and encourage in-depth responses.

- Face-to-face interviews may have encouraged socially desirable responses, by participants exaggerating positive experiences and downplaying negative ones to avoid being overly critical of English antenatal care services in front of the interviewer.

- There is a small, potential risk of selection bias using our method of sampling but nonetheless, the insights gained are likely to be valuable when considering how to improve English antenatal and intrapartum care for Somali women.

non-pregnant women, to align with current guidelines. Women with unfavourable social factors may require additional support to improve access to English antenatal care services.

\section{INTRODUCTION}

Female genital mutilation (FGM), also known as female genital cutting or circumcision, is the partial or complete removal of, or injury to, the external female genitalia for 
non-medical reasons. ${ }^{1}$ The most severe form of FGM is type III (infibulation), which involves the removal and apposition of the inner and outer labia, with or without excision of the clitoris, leading to the creation of a covering seal and narrowing of the vaginal orifice. ${ }^{1}$ Consequences may include haemorrhage, infection or death in the short-term and recurrent urinary tract infections, impaired sexual function and psychological issues in the longer term. ${ }^{1-3}$ Type III FGM also has implications for future childbirth, with women at increased risk of adverse obstetric outcomes including: postpartum haemorrhage, perineal tearing, fistulae, obstructed labour or stillbirth. ${ }^{45}$

FGM affects more than 125 million girls and women worldwide and is reportedly practiced in 29 countries in sub-Saharan Africa and the Middle East. ${ }^{6}$ Recent estimates revealed that 137000 girls and women are living with FGM in England and Wales, although their country of origin was not reported. ${ }^{7}$ In Somalia, a reported $98 \%$ of 15-49-year old females have been affected, mostly by type III $(63 \%){ }^{6}$ There is a large Somali community living in England and Wales $(\mathrm{n}=101370)$, with 9870 (females $=5365$ ) located in the West Midlands. ${ }^{8}$ Given the high prevalence of FGM in Somalia, it is not surprising that the majority (over 90\%) of all pregnant women with FGM accessing specialist antenatal care (ANC) services in Birmingham each year $(n=349)$ are Somali. ${ }^{9} 10$ These women are more likely to present late and delay deinfibulation (reversal of FGM) until the intrapartum period, ${ }^{911}$ which contradicts current guidelines. ${ }^{12}$

Few studies have explored this community's experiences of services in terms of care received, accessibility and cultural sensitivity. A limited number of studies suggest that a lack of health worker knowledge and understanding of FGM undermines confidence and trust in English health services. ${ }^{13}$ This may exacerbate feelings of inadequacy and increase hesitancy of affected women to speak about FGM. ${ }^{14}$ Similar thoughts have been expressed in studies undertaken in Northern America. ${ }^{2} 15$ Health worker knowledge of FGM is especially important for ANC experiences, ${ }^{16}{ }^{17}$ but surveys have shown that health workers lack knowledge ${ }^{1819}$ according to professional guidelines. ${ }^{11}$ For example, FGM is not an absolute indication for caesarean section ${ }^{12}$ yet women are at an increased risk ${ }^{11}$ and may feel pressured by health workers to have one. ${ }^{20}$ Mismanagement of women with FGM during pregnancy and labour may lead to problems at birth, patient dissatisfaction or promote barriers to accessing services in the future. ${ }^{16} 1720$ Therefore, in order to evaluate the acceptability and accessibility of current ANC services, it is important to explore Somali women's experiences further.

The aim of this study therefore was to explore how Somali women exposed to FGM, experience and perceive antenatal and intrapartum care in England by exploring: (1) perceptions of deinfibulation, caesarean section and natural vaginal delivery, (2) experiences of care during pregnancy and labour, and (3) factors that affect ability to access ANC services, in order to make recommendations about future practice.

\section{METHODS}

This exploratory study used descriptive qualitative methodology and is reported against Consolidated criteria for reporting qualitative research (COREQ) guidelines. $^{21}$ Participants were purposively sampled ${ }^{22}$ using the following inclusion criteria: women over the age of 18 ; born in Somalia and resident in the West Midlands; with at least one child under the age of 5 years or were at least 6 months pregnant with their first child at the time of interview; and had the ability to provide informed consent to an audio-recorded interview. Specifically, snowball sampling, a strategy often used in hard-to-reach populations, ${ }^{23}$ and convenience sampling were used. Semistructured interviews were used in order to reconstruct perceptions of ANC experiences and facilitate an in-depth exploration of women's views. ${ }^{24}$ Face-to-face interviews were conducted in a private room, offering women privacy to discuss a potentially sensitive topic.

This study took place in Birmingham, West Midlands. This location was selected due to the large resident Somali community and high numbers of Somali women with FGM accessing ANC services locally. ${ }^{8-10}$ Recruitment and interviews took place between February and April 2015 (interviews started in March) at two community centres, which were identified using a specific recruitment strategy (box 1 ).

Immediately prior to data collection, all participants provided informed verbal consent and interpreter confidentiality agreements were signed as appropriate. Participants were also asked to complete a basic demographic questionnaire following consent. A community outreach worker at centre A reviewed both the topic guide and questionnaire during the initial visit in order to ensure cultural sensitivity. No alterations were made to either document following this review. Semistructured interviews followed a topic guide (table 1), which was informed by both a review of the literature, advice from academics experienced in Somali community research and discussion between the authors. The guide covered a variety of prespecified topics but remained flexible, allowing women to highlight additional issues that were unforeseen at the study outset. ${ }^{28}$ Using an iterative approach, ${ }^{24}$ the first four interviews were reviewed and after this, the topic guide was expanded to include further topics identified by the participants. Interviews lasted an average of $20 \mathrm{~min}$ (range 6-38 $\mathrm{min}$ ). A lay female interpreter, identified and trusted by the community, was present in five out of the seven interviews at centre B but only actively translated in three. No interpreters were used at centre A. Interviews were audio-recorded with consent. Brief field notes were taken during the interviews and reflected on immediately. All participants received an inconvenience allowance in the form of a $£ 10$ shopping voucher at the end of the interview. 


\section{Box 1 Summary of recruitment strategy}

Recruitment was expected to be challenging due to difficulties engaging the Somali community for research purposes ${ }^{25}$ and the potentially sensitive nature of the research question. As an outsider to the community, a combination of recruitment techniques was required in order to facilitate the identification of community stakeholders and gatekeepers. ${ }^{26}$ Using previous insights into ethnic minority research, ${ }^{27}$ a collaborative recruitment strategy was devised. The strategy was as follows:

1. Identify community centres, stakeholders and gatekeepers using:

A. Internet search engines

B. The Birmingham City Council website

C. Social networks of the researchers

2. Make initial contact (email or telephone) with candidate centres personally or alternatively, via a stakeholder working in collaboration (using pre-established relationships to gain the community's trust)

3. Provide willing centres with full details of the study and devise a plan for recruitment

Centre A (children's centre) was identified from Birmingham City Council and centre B (community centre) through a stakeholder (community member) identified from JMM's social network. During an initial visit at centre A, JMM provided copies of the Participant Information Sheet (PIS) to the local community outreach worker who, subsequently, contacted and recruited women $(n=3)$ into the study using convenience sampling methods. At centre B, the stakeholder was provided with the PIS and communicated this to the centre manager. The manager and a colleague recruited participants in advance of and during JMM's first visit using convenience $(n=1)$ and snowball $(n=6)$ sampling methods. Sampling ended when no further participants could be identified.

Data were analysed using inductive thematic analysis, ${ }^{29}$ with codes and subsequent themes being generated from the data itself rather than using an a priori coding frame. The analytic process was iterative and took place concurrently with data collection. Audio recordings were transcribed clean verbatim by JMM to improve narrative flow. ${ }^{30}$ Transcripts were read repeatedly to aid familiarisation and allow for data immersion. This facilitated the generation of preliminary codes and themes using NVivo software, which eventually progressed into a developed coding frame. Two of the most concept-rich transcripts were independently double-coded by LLJ and additional interpretations were incorporated into the coding frame. Once all interviews had been coded, data were organised into a framework in accordance with codes and themes, in order to visualise the data set in a systematic way. Divergent cases were explored and reported in the findings where appropriate. The final themes were agreed by both authors and have been summarised in table 2.

\section{RESULTS}

In total, 10 women were interviewed. A summary of participant demographics is shown in table 3 . Three main themes were interpreted within the data: (1)
Table 1 Summary of interview topic guide

\begin{tabular}{|c|c|}
\hline Topic area & Summary of subtopics covered \\
\hline $\begin{array}{l}1 \text { Antenatal care } \\
\text { experiences }\end{array}$ & $\begin{array}{l}\text { Exploration of a woman's } \\
\text { experiences and perceptions of } \\
\text { antenatal care services, } \\
\text { specifically: } \\
\text { The relationship between the } \\
\text { woman and the midwife } \\
\text { The midwife's awareness of } \\
\text { Somali culture, religion and } \\
\text { FGM } \\
\text { Thoughts, ideas and opinions } \\
\text { about deinfibulation and the } \\
\text { appropriate time to undergo it }\end{array}$ \\
\hline 2 Labour experiences & $\begin{array}{l}\text { Exploration of a woman's } \\
\text { experiences of labour and } \\
\text { delivery, specifically: } \\
\text { Thoughts, feelings and } \\
\text { experiences of care during } \\
\text { labour } \\
\text { Sources of support during } \\
\text { labour, for example, relatives, } \\
\text { friends, healthcare professionals } \\
\text { Perceptions and/or } \\
\text { experiences of vaginal delivery } \\
\text { and caesarean section }\end{array}$ \\
\hline $\begin{array}{l}3 \text { Access to women's } \\
\text { health services }\end{array}$ & $\begin{array}{l}\text { Exploration of enablers of and } \\
\text { barriers to presenting to antenatal } \\
\text { care services and suggestions for } \\
\text { future improvement to services }\end{array}$ \\
\hline
\end{tabular}

experiences of FGM during life, pregnancy and labour, (2) experience of care from midwives, (3) adaptation to English life.

\section{Experiences of FGM during life, pregnancy and labour Knowledge of and personal beliefs about FGM}

The majority of participants used the term 'circumcision' (P1, P3, P5, P6) when describing FGM but other terms used included 'FGM' (P8, P9) or 'cutting' (P7). The remaining participants referred to FGM as 'it'. Women displayed varying levels of knowledge around FGM but generally, did not describe it in detail. Despite this, the majority expressed strong personal beliefs about the practice. One woman was both aware of and in agreement with the illegality of FGM in England: "they come here and they will end in the prison that's good because you hurt someone" (P4). In addition, beliefs appeared to be informed by women's experiences of FGM and its sequelae, with the latter cited as a strong reason against subjecting their daughters to it:

It's cruelty actually doing it and I always get asked,

"would you actually do it to, you know, to your girls" and I'm like "no way! No, of course not." Yeah. Because it ruins everything. (P9) 
Table 2 Summary of interpreted themes and subthemes

\begin{tabular}{|c|c|}
\hline Theme & Subtheme \\
\hline $\begin{array}{l}1 \text { Experiences of FGM } \\
\text { during life, pregnancy } \\
\text { and labour }\end{array}$ & $\begin{array}{l}\text { 1. Knowledge of and } \\
\text { personal beliefs about } \\
\text { FGM } \\
\text { 2. Impact of FGM on life } \\
\text { 3. Impact of FGM during } \\
\text { pregnancy and labour } \\
\text { 4. Experiences and } \\
\text { perceptions of } \\
\text { deinfibulation or reversal } \\
\text { 5. Mode and location of } \\
\text { delivery }\end{array}$ \\
\hline $\begin{array}{l}2 \text { Experience of care from } \\
\text { midwives }\end{array}$ & $\begin{array}{l}\text { 1. Relationship between the } \\
\text { woman and the midwife } \\
\text { 2. Midwife awareness of } \\
\text { Somali culture and } \\
\text { religion }\end{array}$ \\
\hline 3 Adaptation to English life & $\begin{array}{l}\text { 1. Cultural beliefs and } \\
\text { perceptions about ANC } \\
\text { services } \\
\text { 2. Ability to access ANC } \\
\text { services }\end{array}$ \\
\hline
\end{tabular}

\section{Impact of FGM on life}

Most women described FGM as a traumatic experience that had had a lasting impact on their physical and psychological well-being. Physical problems included difficulty having sexual intercourse: “...because it was closed it was stitched up so I could only go toilet apart anything else you know wouldn't ever go in, in there so..." (P9), difficult menstruation and increased pain: "it's when normal monthly period I know people suffer from that. They have lots more pain than normal people do" (P8). Psychological issues included fear, worry or embarrassment: "I was always you know embarrassed of it because I thought it was strange, you know, why are you scared?" (P9). This observation was not entirely universal, as one woman expressed deviating views regarding the trauma of FGM and its subsequent impact on later life: "It was a traumatic experience but it hasn't really affected my life afterwards" (P5).

\section{Impact of FGM during pregnancy and labour}

The influence of FGM was apparent in all affected women, regardless of whether they had suffered obstetric complications. At a minimum, women reported preexisting expectations of having problems during labour having received warnings from older Somali women or health workers. Moreover, pregnancy appeared to exacerbate pre-existing psychological issues, such as a fear of giving birth:

I thought of her actually coming through out you know from there. I thought it was strange and awkward, would I you know bleed, I dunno, I was scared of it because of
Table 3 Participant demographic summary

\begin{tabular}{|c|c|}
\hline Demographic characteristic & $\mathbf{n}$ \\
\hline \multicolumn{2}{|l|}{ Age group (years) } \\
\hline 20-29 & 2 \\
\hline 30-39 & 5 \\
\hline $40-49$ & 3 \\
\hline \multicolumn{2}{|l|}{ Employment status } \\
\hline Employed & 3 \\
\hline Unemployed & 7 \\
\hline \multicolumn{2}{|l|}{ Marital status } \\
\hline Married & 9 \\
\hline Unmarried & 1 \\
\hline \multicolumn{2}{|l|}{ Number of years in England } \\
\hline $6-10$ & 5 \\
\hline $11-15$ & 2 \\
\hline$>20$ & 3 \\
\hline \multicolumn{2}{|l|}{ Number of children } \\
\hline Less than 5 & 4 \\
\hline 5 or more & 6 \\
\hline \multicolumn{2}{|l|}{ Pregnancy status } \\
\hline Pregnant & 0 \\
\hline Non-pregnant & 10 \\
\hline \multicolumn{2}{|l|}{ Location of interview } \\
\hline Centre A & 3 \\
\hline Centre B & 7 \\
\hline \multicolumn{2}{|l|}{ Interpreter required } \\
\hline Centre A & 0 \\
\hline Centre B & 3 \\
\hline \multicolumn{2}{|l|}{ Undergone FGM ${ }^{*}$} \\
\hline Yes & 8 \\
\hline No & 2 \\
\hline
\end{tabular}

*All women voluntarily disclosed FGM status, without prior prompting from the interviewer.

FGM, female genital mutilation.

the, you know because it was closed for so many [years] ...it was terrifying yeah. (P9)

The physical impact of FGM during pregnancy was alluded to through reports of increased pain (P3-P5), infection (P6) and perineal tearing (P9). These complications, described as 'difficult and traumatic' (P6) and 'so horrible' (P9), tended to make the overall experience of labour more negative.

\section{Experiences and perceptions of deinfibulation or reversal}

Generally, women reported positive experiences and easier births after deinfibulation. However, despite repairing the physical problem, deinfibulation did not always address the psychological impact with one woman reporting a fear of intimate examinations: "In terms of was it fixed in my, you know, mind, it's not at all...even with my smear test I struggle still, I'm scared of it" (P9). Most women preferred to be deinfibulated during the intrapartum rather than antenatal period due to a reluctance to undergo two invasive procedures. In particular, women that anticipated needing an episiotomy delayed deinfibulation until labour in order to ensure that any surgical procedures were done at the same time: "I can't 
have two operations...so I chose it [deinfibulation] when I have a baby" (P2). In contrast to the others, one woman (P9) underwent deinfibulation before pregnancy. This was done in order to ease the physical difficulties associated with FGM such as problems with sexual intercourse. She reported that many newlywed women from the community do not recognise this as an option. Instead, women 'accept' the belief that the stitched area must be 'opened' by the husband in order to get pregnant, using force and/or instruments if necessary.

Apparently the husband he forces...to actually, you know, get it opened. He, I heard they actually use instruments, as in a blade...their thinking is if I don't open my, what's it, wife, I'm not man enough for anything...It's almost as if it's a pride. (P9)

\section{Mode and location of delivery}

The majority of participants agreed that giving birth in hospital was 'safer' (P10) than delivering at home. All women gave birth to at least one child by vaginal delivery and two women (P4, P9) underwent caesarean section. Vaginal delivery was seen as 'normal' (P2) or 'natural' (P1) and, generally, the preferred method of delivery. There were mixed opinions about undergoing caesarean section. The majority of women felt that caesarean section was not natural and would only consider it as a last resort: "I don't see as natural and so if my health is at risk, then I would go for C-section but to start with there's no point really" (P10). Factors influencing decisions about mode of delivery included previous birth experiences, experiences of significant peers and perceptions about medical advances. In addition, psychological trauma from previous experiences strongly affected the decision-making process:

I thought because of the first experience, it was so horrible, I thought I could have a C-section, you know, I thought there's a chance of the tearing, you know, once again...I mean I kind of regret it. I wish I had a, you know, normal [vaginal delivery] because I assumed oh a caesarean easier but only it's not easier. It's horrible! (P9)

\section{Experience of care from midwives}

\section{Relationship between the woman and the midwife}

The relationship between the woman and the midwife appeared to be fundamental to a woman's experience of care, with better relationships resulting in more positive experiences. The majority of women had open relationships with their midwives and were comfortable discussing FGM, particularly when the midwife initiated the conversation. When women did not have an open relationship, they felt unable to discuss FGM and were not able to communicate their concerns:

I kept it [FGM] hidden because I thought it was kind of like embarrassing...so I didn't tell her, I didn't say I was scared or anything. I said, “yeah everything's okay, yeah
I'm fine with it" but you know when it was actually happening it was a different story. (P9)

In contrast, there were mixed opinions about the care received by midwives during labour. Relationships underpinned by a lack of communication resulted in more negative experiences. One woman (P4) was told to wait in a corridor for over 90 min by midwives, despite being in severe pain and receiving no explanation. Another woman reported a negative labour experience where she was under the supervision of student midwives:

\begin{abstract}
...They were only student, so it was all like confusing you know whether in terms of the pushing stage. I wasn't told you know when to stop, so I had a third degree tear so I was like, it was just like because they were students I feel like they weren't as experienced as a real professional...It wasn't what I expected. I expected for a midwife to tell me because it was a first you know first baby. (P9)
\end{abstract}

\section{Midwife awareness of Somali culture and religion}

Midwife awareness of patient culture and religion was key to developing good professional relationships. Midwives were reported to have good awareness about cultural and religious issues and phrased questions about FGM in a culturally sensitive way. Women did not mind being asked about their views on FGM and whether they would subject their daughters to it.

No I don't got problem with that if they ask me about yeah the questions it's our culture, it's normal for us you know. (P1)

Additionally, women reported that midwives gave them treatment options that would respect their cultural and religious beliefs. For example, women were able to choose when to undergo deinfibulation (P2, P3), which midwife they wanted during pregnancy (P8), whether they wanted a caesarean section (P9) and whether they would like to be seen by only female midwives (P6-P10). Most women, except for one participant (P5), preferred to be seen by a female midwife. This was due to religious and/or personal reasons, such as not wanting a man to see their private area.

\section{Adaptation to English life \\ Cultural beliefs and perceptions about ANC services}

Overall, participants were satisfied with current English ANC services. Some concerns were highlighted, such as a perceived lack of information or understanding about how ANC services work. One woman described how, before she came to England, she was warned that her child could be stolen from hospital. She reported that after delivery she was: "constantly looking at the child during, just worried that someone was going to take the child" (P5). In addition, health workers had told her to 'look after her child', which reinforced her fears. While other participants did not believe that child stealing or switching occurred in England, one woman suggested 
reasons why the rumour remains prevalent in the community:

I feel like as a community we get very worried about things because we don't really know much about the antenatal care services in England, we don't know how things work here. I have heard those rumours and it is something that is mentioned a lot within the community. (P6)

\section{Ability to access ANC services}

Women did not report difficulty in accessing ANC services. Many had lived in England for a long time, had advanced language skills or, if not, had good social support networks, from which a lay interpreter could be identified. One woman (P1) did not perceive it to be difficult for someone to access services but others (P7) used past experiences of being new to England to reflect on the challenges that new migrants might face. Many agreed that 'language is really the key to everything' (P8). Without language, women were unable to find information, to communicate problems or needs, or be understood by health workers. In addition, certain social factors such as driving ability, financial situation and marital status were thought to hinder a woman from accessing ANC services.

\section{DISCUSSION}

This study explored the views of 10 Somali women exposed to FGM on their experiences and perceptions of antenatal and intrapartum care. Findings showed that FGM had a significant impact on women's experiences of pregnancy and labour, with midwives highlighted as having an important role in shaping overall experiences and perceptions of antenatal and intrapartum care.

The high prevalence of FGM among Somali women was reflected in this study, as $80 \%$ of participants reported they had undergone FGM, despite the fact we did not purposively recruit women with FGM nor was their FGM status known prior to interview. Obstetric complications such as perineal tearing were reported by the participants and psychological trauma, particularly fear of giving birth and aversion to intimate medical examinations, was also apparent. Feelings of fear may influence a woman's decision about how she would like to give birth. All women preferred to give birth in hospital, as it was perceived as safer, but there were mixed opinions about choosing vaginal or caesarean delivery. Guidelines state that FGM is not an indication for caesarean section ${ }^{11}$ but a woman in this study chose it regardless. It is important to distinguish between women who request caesarean section out of fear rather than as an informed choice, as those who choose it out of fear may later express feelings of regret as evidenced in the current study.

It is recommended that deinfibulation be performed before pregnancy or around 20 weeks gestation to minimise the risk of the fetal head causing extensive perineal tearing and avoid the need to cut scar tissue in labour. ${ }^{12}$ A previous study suggested that these recommendations are not being followed, with a reported $77 \%$ of infibulated women choosing intrapartum deinfibulation. ${ }^{9}$ Findings from this study may help explain this, as women who are anticipating having an episiotomy preferred to delay deinfibulation until labour to avoid undergoing two separate surgical interventions. In addition, non-pregnant women may not seek deinfibulation services at all due to a lack of awareness or a cultural belief that the stitched area should be 'opened' by the husband. One participant reported that men might use force or instruments such as blades to do this, which may put women in danger of excess blood loss and infection. However, this has not previously been identified in the current evidence base, and so the pervasiveness of this practice among the Somali population in England is currently unknown.

As shown in this study, midwife awareness and knowledge of FGM is an important factor in providing adequate care to affected women. Midwives are central in Somali women's experiences of antenatal and intrapartum services, with better relationships resulting in more positive experiences. Most women are comfortable discussing FGM and have previously reported higher satisfaction levels when treated by health workers with prior knowledge of it. ${ }^{16}{ }^{17}$ This supports the findings of this study, which show women's experiences are influenced by midwives' awareness of FGM, their willingness to discuss FGM and the support provided around issues caused by FGM. However, midwife awareness of FGM appears to vary across England. An audit undertaken at a major teaching hospital in inner London showed that, despite access to a specialist African Women's Clinic for women with FGM, only $54 \%$ of women with FGM were identified by midwives at the booking visit. ${ }^{31}$ In contrast, a hospital midwifery-led FGM service in Birmingham identified $88.5 \%$ of affected women at booking. ${ }^{9}$ All midwives should be aware that Somali women are at a higher risk of FGM and should be routinely asked about this at booking. It is possible that, despite having awareness of FGM, midwives lack confidence to broach the subject due to insufficient knowledge, experience and understanding of patient culture. ${ }^{32}$ It is important to explore this further and identify areas of improvement, as mismanagement of FGM during the antenatal period may increase the risk of birth problems ${ }^{20}$ or cause psychological harm.

Adaptation to English life highly influences women's ability to access ANC services. The Saving Mothers' Lives report $^{33}$ showed that $10 \%$ of maternal deaths between 2003 and 2005 were in non-English-speaking migrants. Of these, $35 \%$ of women were late or non-attenders to ANC services. Women in our study helped explain this by highlighting enablers of and barriers to access. Ability to speak English was perceived as the key factor in enabling a pregnant woman to obtain the care she needs but, in addition, other social factors, such as number of young children, marital status, financial situation, proximity of health services and ability to drive or use public 
transport, were also deemed important. Ability to access services appears to improve as women spend longer in England, as their English skills improve and as they develop social support networks with more experienced migrants. Social support networks provide them with guidance and stability and, as a result, many women could be influenced by the views of other community members. This may have a positive or negative effect, by encouraging women at risk to present or discouraging them if services have developed a bad reputation through the perpetuation of rumours.

\section{STRENGTHS AND LIMITATIONS}

This qualitative study, as far as the authors are aware, is the first to describe how Somali women exposed to FGM experience and perceive antenatal and intrapartum care in England. Although the sample size was relatively small which may limit the transferability of the findings, the insights gained may be important when considering how to optimise antenatal and intrapartum care for Somali women. In order to overcome the challenges of recruitment, community gatekeepers were asked to approach potential participants and recruit women by convenience sampling. Consequently, it was not possible to record response rates or demographic differences between those who elected to participate and those who did not. Without this information, the possibility of selection bias cannot be ruled out. Another limitation was the use of lay interpreters, which may have promoted misinterpretation of questions and responses during interview due to insufficient linguistic ability and research experience. ${ }^{34}$ Additionally, due to the limited time frame of the study, the effect of the interpreter on the data was not accounted for, which may have compromised cross-language trustworthiness of the translated data. ${ }^{35}$ Despite this, using trusted interpreters appeared to facilitate the development of rapport, put participants at ease and encourage in-depth responses. This may not have occurred had the interpreter been externally sourced. The background of the interviewer (non-Somali, medical student, middle-class, no children) may have inadvertently influenced the line of questioning and interpretation of the data during interview and analysis. Therefore, investigator triangulation (independent double-coding) was utilised to increase the reliability of the findings. ${ }^{36}$ Additionally, face-to-face interviews may have encouraged socially desirable responses, by participants exaggerating positive experiences and downplaying negative ones to avoid being overly critical of ANC services in front of the interviewer. ${ }^{37}$ The impact of this was considered to be small as participants reported a wide range of views.

\section{CLINICAL AND RESEARCH RECOMIMENDATIONS}

FGM has a significant impact on Somali women's experiences of antenatal and intrapartum care in England. For this reason, it is important that pregnant women with
FGM are identified early and assigned to an experienced and culturally aware midwife, where possible. A positive and open relationship must be established early in pregnancy to facilitate discussions about FGM, including need for deinfibulation, plans for delivery, possible birth complications and child safeguarding. These discussions cannot be ignored, as failure to address patient concerns may increase the risk of birth complications and/or cause additional psychological harm. Most women were comfortable discussing these issues but may be reluctant to initiate the conversation. Therefore, this study recommends that midwives make it routine practice to ask all pregnant Somali women about FGM at the booking visit. This is especially important given the high prevalence of FGM in Somalia. Assessing midwives' feelings about this is an important area for future research, in order to establish whether additional training or guidance is required.

The findings of this study support previous evidence demonstrating the unpopularity of antenatal deinfibulation among infibulated Somali women. ${ }^{9}$ Guidelines recommend an alternative, pre-pregnancy deinfibulation, but women may not be aware of this procedure before they access ANC services. As women may be at risk of forceful deinfibulation within the community, it is important to increase awareness of deinfibulation before they attempt to conceive, as this is safer and will align with current guidelines. ${ }^{11}{ }^{12}$ In order to determine how best to raise awareness, the health literacy of Somali women should be taken into consideration. This study found that women who were less adapted to English life, in terms of language skills, knowledge of how services work and strength of social support networks, were more likely to have difficulty accessing ANC services, to believe speculative rumours and to have negative experiences. Language barriers are well recognised as a cause for poor health literacy; ${ }^{38}$ therefore, a well-designed and appropriately targeted national education campaign, to increase community knowledge and improve access to ANC services, is indicated. Historietas (comic book-style health education brochures) have been validated by Somali women as useful tools to increase knowledge about emergency caesareans ${ }^{38}$ and could be applied similarly to increase knowledge about how ANC services work or to demonstrate the benefits of pre-pregnancy deinfibulation to women at risk of forceful deinfibulation within the community.

Acknowledgements The authors thank all participating women and community centres, as well as Haweya Abdikadir and Zabina Qousar for their help facilitating recruitment. In addition, the authors thank Dr Gilles de Wildt, Dr Jonathan Ives, Professor Heather Draper and Dr Naomi Tomlinson for their professional guidance and Julie Shore and Lucy Milton for providing logistical support during this study.

Contributors JMM conceived the study and designed it in collaboration with LLJ. JMM conducted and transcribed the interviews. Data were coded by JMM and independently double-coded by LLJ. JMM and LLJ subsequently reviewed all coded data together and agreed on a final coding framework. JMM wrote the initial drafts of the manuscript, which were reviewed, edited and amended by LLJ. The final version of the manuscript was approved by both authors. 
Funding This work was supported by a grant from the Sir Arthur Thomson Trust at the University of Birmingham.

Competing interests None declared.

Ethics approval This study received a favourable ethical opinion from the University of Birmingham Internal Ethics Review Committee (Ref: 2014-5/C2/ DK/06).

Provenance and peer review Not commissioned; externally peer reviewed.

Data sharing statement No additional data are available.

Open Access This is an Open Access article distributed in accordance with the Creative Commons Attribution Non Commercial (CC BY-NC 4.0) license, which permits others to distribute, remix, adapt, build upon this work noncommercially, and license their derivative works on different terms, provided the original work is properly cited and the use is non-commercial. See: http:// creativecommons.org/licenses/by-nc/4.0/

\section{REFERENCES}

1. World Health Organisation. Female genital mutilation. 2004. http:// www.who.int/mediacentre/factsheets/fs241/en/ (accessed 4 May 2015).

2. Chalmers B, Hashi KO. 432 Somali women's birth experiences in Canada after earlier female genital mutilation. Birth 2000;27:227-34.

3. Behrendt A, Moritz S. Posttraumatic stress disorder and memory problems after female genital mutilation. Am J Psychiatry 2005;162:1000-2.

4. Rushwan $\mathrm{H}$. Female genital mutilation (FGM) management during pregnancy, childbirth and the postpartum period. Int J Gynaecol Obstet 2000;70:99-104.

5. Banks E, Meirik O, Farley T, et al. Female genital mutilation and obstetric outcome: WHO collaborative prospective study in six African countries. Lancet 2006;367:1835-41.

6. United Nations Children's Fund. Female genital mutilation/cutting: a statistical overview and exploration of the dynamics of change. 2013. http://www.childinfo.org/files/FGCM_Lo_res.pdf (accessed 8 Nov 2014).

7. Macfarlane AJ, Dorkenoo E. Estimating the numbers of women and girls with female genital mutilation in England and wales. J Epidemiol Community Health 2015;69:A61.

8. Office for National Statistics. 2011 Census: QS203EW Country of birth (detailed), local authorities in England and Wales. 2011. http:// www.ons.gov.uk/ons/rel/census/2011-census/

key-statistics-for-local-authorities-in-england-and-wales/ rft-table-qs203ew.xls (accessed 9 Nov 2014).

9. Paliwal P, Ali S, Bradshaw S, et al. Management of type III female genital mutilation in Birmingham, UK: a retrospective audit. Midwifery 2014;30:282-8.

10. Heart of England NHS Foundation Trust. FOI 3023 FGM. http://www. heartofengland.nhs.uk/foi-3023-fgm/ (accessed 25 Apr 2015).

11. RCOG. Female Genital Mutilation and its Management. Green-top Guideline. May No. 53. 2009. https://www.rcog.org.uk/globalassets/ documents/guidelines/greentop53femalegenitalmutilation.pdf (accessed 21 Oct 2014)

12. RCN. Female genital mutilation: an RCN resource for nursing and midwifery practice. 2nd edn. 2015. http://www.rcn.org.uk/_data/ assets/pdf_file/0010/608914/RCNguidance_FGM_WEB2.pdf (accessed 21 Apr 2015)

13. Hussein E. Women's experiences, perceptions and attitudes of female genital mutilation: the Bristol PEER study. 2010. http://www. forwarduk.org.uk/wp-content/ uploads/2014/12/ Womens-Experiences-Perceptions-and-Attitudes-of-FemaleGenital-Mutilation-The-Bristol-PEER-Study.pdf (accessed 26 Oct 2015).

14. Oguntoye S, Otoo-oyortey N, Hemmings J, et al. FGM is with us everyday women and girls speak out about female genital mutilation in the UK. World Acad Sci Eng Technol 2009;30:1015-20.
15. Ameresekere M, Borg R, Frederick J, et al. Somali immigrant women's perceptions of cesarean delivery and patient-provider communication surrounding female circumcision and childbirth in the USA. Int J Gynaecol Obstet 2011;115:227-30.

16. Vangen S, Johansen RE, Sundby J, et al. Qualitative study of perinatal care experiences among Somali women and local health care professionals in Norway. Eur J Obstet Gynecol Reprod Biol 2004;112:29-35.

17. Lundberg PC, Gerezgiher A. Experiences from pregnancy and childbirth related to female genital mutilation among Eritrean immigrant women in Sweden. Midwifery 2008;24:214-25.

18. Zaidi N, Khalil A, Roberts C, et al. Knowledge of female genital mutilation among healthcare professionals. J Obstet Gynaecol 2007;27:161-4.

19. Purchase TC, Lamoudi M, Colman S, et al. A survey on knowledge of female genital mutilation guidelines. Acta Obstet Gynecol Scand 2013;92:858-61.

20. Straus L, McEwen A, Hussein FM. Somali women's experience of childbirth in the UK: perspectives from Somali health workers. Midwifery 2009;25:181-6.

21. Tong A, Sainsbury P, Craig J. Consolidated criteria for reporting qualitative research (COREQ): a 32-item checklist for interviews and focus groups. Int J Qual Health Care 2007;19:349-57.

22. Patton M. Qualitative evaluation and research methods ( pp. 169186): purposive sampling. 1990. http://legacy.oise.utoronto.ca/ research/field-centres/ross/ctl1014/Patton1990.pdf (accessed Nov 2014).

23. Penrod J, Preston DB, Cain RE, et al. A discussion of chain referral as a method of sampling hard-to-reach populations. J Transcult Nurs 2003;14:100-7.

24. Dicicco-Bloom B, Crabtree BF. The qualitative research interview. Med Educ 2006;40:314-21.

25. Hemmings J. Understanding East London's Somali Communities: a study conducted for the East London Alliance. 2010. http:// karin-ha.org.uk/wp-content/uploads/2013/02/UnderstandingEast-Londons-Somali-Communities.pdf (accessed 25 Apr 2015).

26. Sixsmith J, Boneham M, Goldring JE. Accessing the community gaining insider perspectives from the outside. Qual Health Res 2003;13:578-89.

27. Elam G, Fenton KA. Researching sensitive issues and ethnicity: lessons from sexual health. Ethn Health 2003;8:15-27.

28. Al-Busaidi ZQ. Qualitative research and its uses in health care. Sultan Qaboos Univ Med J 2008;8:11-19.

29. Braun V, Clarke V. Using thematic analysis in psychology. Qual Res Psychol 2006;3:77-101.

30. Bailey J. First steps in qualitative data analysis: transcribing. Fam Pract 2008;25:127-31.

31. Zenner N, Liao L, Richens Y, et al. Quality of obstetric and midwifery care for pregnant women who have undergone female genital mutilation. J Obstet Gynaecol 2013;33:459-62.

32. Dawson AJ, Turkmani S, Varol N, et al. Midwives' experiences of caring for women with female genital mutilation: insights and ways forward for practice in Australia. Women Birth 2015;28:207-14.

33. Lewis G. The Confidential Enquiry into Maternal and Child Health $(\mathrm{CEMACH})$. Saving Mothers' Lives: reviewing maternal deaths to make motherhood safer 2003-2005, the seventh report on confidential enquiries into maternal deaths in the United Kingdom. 2007. http://www.publichealth.hscni.net/sites/default/files/Saving \%20Mothers'\%20Lives\%202003-05\%20.pdf (accessed 4 May 2015)

34. Kapborga I, Bertero C. Using an interpreter in qualitative interviews: does it threaten validity? Nurs Inq 2002;9:52-6.

35. Squires A. Methodological challenges in cross-language qualitative research: a research review. Int J Nurs Stud 2009;46:277-87.

36. Denzin NK. The research act in sociology. Chicago: Aldine, 1970.

37. Neeley SM, Cronley ML. When research participants don't tell it like it is: pinpointing the effects of social desirability bias using self vs. Indirect-questioning. Adv Consum Res 2004;31:432-3.

38. Jacoby SD, Lucarelli M, Musse $F$, et al. A mixed-methods study of immigrant Somali women's health literacy and perinatal experiences in Maine. J Midwifery Womens Health 2015;60:593-603. 